(C) 2018, The Authors. Published by FASS Inc. and Elsevier Inc. on behalf of the American Dairy Science Association ${ }^{\circledR}$. This is an open access article under the CC BY-NC-ND license (http://creativecommons.org/licenses/by-nc-nd/4.0/).

\title{
Size, shape, and identity of surface crystals and their relationship to sensory perception of grittiness in soft smear-ripened cheeses
}

\author{
P. J. Polowsky, ${ }^{*}$ P. S. Kindstedt, ${ }^{* 1}$ and J. M. Hughes $\dagger$ \\ *Department of Nutrition and Food Sciences, and \\ †Department of Geology, University of Vermont, Burlington 05405
}

\begin{abstract}
Soft smear-ripened cheeses undergo extensive surface crystallization and radial demineralization of calcium, magnesium, and phosphorus, which likely contributes to radial softening during ripening. Furthermore, anecdotal evidence suggests that grittiness is a common characteristic of smear-ripened cheeses. The primary aims of the present study were to evaluate the intensity of perceived grittiness while assessing other key sensory attributes in US artisanal and European protected designation of origin smear-ripened cheeses, and to relate perceived grittiness to the size, shape, and identity of crystals present in the cheese surface smears. Fully ripened wheels of 24 different varieties of smear-ripened cheeses, 16 produced in the United States and 8 in the European Union, were obtained from retail sources. A trained sensory panel $(\mathrm{n}=12)$ was employed to evaluate intensity of grittiness. Crystals present in the cheese smears were identified by powder X-ray diffractometry and polarized light microscopy, and further evaluated in polarized light microscopy micrographs by image analysis for size and shape characteristics. Mean sensory scores for the 24 cheeses ranged from no perceived grittiness to easily identifiable grittiness. Surface crystals included ikaite, struvite, calcite, and brushite, and mean crystal length and area ranged among cheeses from 27 to $1,096 \mu \mathrm{m}$, and 533 to $213,969 \mu \mathrm{m}^{2}$, respectively. Panel threshold for grittiness occurred at a mean crystal length of about $66 \mu \mathrm{m}$ and mean crystal area of about 2,913 $\mathrm{\mu m}^{2}$. Cheeses with mean values at or below these thresholds displayed negligible perceived grittiness. In contrast, for cheeses with mean values above these thresholds, the mean sensory scores for grittiness were highly correlated with mean crystal length and crystal area $(r=0.93$ and 0.96 , respectively). Results suggest that surface crystals in soft smear-ripened cheeses influence sensory perception of texture in complex ways that likely include radial softening and grit-
\end{abstract}

Received June 5, 2018.

Accepted August 2, 2018

${ }^{1}$ Corresponding author: Paul.Kindstedt@uvm.edu tiness development. A better understanding of factors that govern surface crystal formation may lead to improved control over crystallization and more consistent cheese texture.

Key words: cheese, surface smear, crystals, grittiness

\section{INTRODUCTION}

Soft smear-ripened cheeses, also known as soft washed-rind cheeses, can encompass a wide variety of products. Several underlying commonalities, however, are the surface application of a brine solution during aging, the resulting outgrowth and proliferation of a complex surface microbiota, and the radial softening or liquefaction of the cheese body over time (Brennan et al., 2004). These actions bring about the characteristic reddish-orange appearance, soft texture, and distinctive flavor that many associate with soft smear-ripened cheeses.

The surface ecologies of smear-ripened cheeses are complex and can contain dozens of genera of fungi and bacteria, such as Penicillium, Debaryomyces, Brevibacterium, Corynebacterium, and many others (Wolfe et al., 2014; Dugat-Bony et al., 2016). These microbes, and their associated metabolisms, can have dramatic effects on cheese chemistry and ripening. The metabolization of lactate and subsequent $\mathrm{pH}$ rise can induce precipitation and crystallization of minerals at the cheese surface, causing radial migration of minerals outward (Tansman et al., 2017a). A homologous process has been observed in a closely related cheese variety, white mold cheeses, such as Brie and Camembert (Le Graet et al., 1983; Le Graet and Brule, 1988; Tansman et al., 2017b). Another consequence of surface microbe activity is the production of gaseous metabolites such as carbon dioxide and ammonia (Karahadian and Lindsay, 1987; Riahi et al., 2007). These alkaline surface conditions, combined with the aforementioned minerals and gaseous components, result in various surface-situated crystals formed on washed-rind cheeses. Ikaite (calcium carbonate hexahydrate, $\mathrm{CaCO}_{3} \cdot 6 \mathrm{H}_{2} \mathrm{O}$ ), calcite (calcium carbonate, $\mathrm{CaCO}_{3}$ ), struvite (magnesium ammonium phosphate hexahydrate, $\left.\mathrm{NH}_{4} \mathrm{MgPO}_{4} \cdot 6 \mathrm{H}_{2} \mathrm{O}\right)$, and brush- 
ite (dicalcium phosphate dihydrate, $\mathrm{CaHPO}_{4} \cdot 2 \mathrm{H}_{2} \mathrm{O}$ ) have been previously identified on soft washed-rind cheeses (Tansman et al., 2017a,b).

Although the identity of these crystals have been confirmed using a robust set of methodologies including powder X-ray diffractometry (PXRD), single crystal X-ray diffractometry, and polarized light microscopy (PLM), their incidence have only been confirmed in a small number of cheeses from a few producers (Tansman et al., 2015, 2017a,c; Polowsky et al., 2018). Moreover, the direct effect of these crystals on the sensorial properties of washed-rind cheeses remains an unresolved question. Informal reports from cheesemakers, cheese mongers, and nonscholarly publications indicate that a relatively common defect of this cheese variety is a gritty or sandy mouthfeel associated with the rind (M. Kehler, Cellars at Jasper Hill, Greensboro, VT, personal communication). We found only one mention in scientific literature referencing this proposedly common defect (Le Mens et al., 2000), but none studying its possible linkage to surface crystals. The present study addresses this gap in knowledge. Previous work demonstrated that surface crystals on washed-rind cheeses can grow to large sizes, on the order of hundreds of micrometers (Tansman et al., 2017a; Polowsky et al., 2018). This size of crystal most likely exceeds the sensory perception threshold, which has been reported in food products to be anywhere from $\sim 20$ to $105 \mu \mathrm{m}$ depending on product and number of crystals present (Vaisey-Genser et al., 1989; Hough et al., 1990; Goff and Hartel, 2013). A systematic study comparing crystal size to grittiness occurrence in smear-ripened cheese is needed to confirm what sensorial effects these crystals contribute to this cheese variety.

Looking broadly, the occurrence of calcium lactate crystals in Cheddar cheese (Johnson et al., 1990; Agarwal et al., 2006; Tansman et al., 2015) and AA crystals in Parmesan-style cheeses (Tansman et al., 2015; D'Incecco et al., 2016) are well known in the dairy technology world, and their effects on cheese quality have been studied to some extent. Such work has yet to be done in smear-ripened cheeses. The specific objectives of the present study were to (1) evaluate grittiness and other key sensory attributes in smear-ripened cheeses and (2) to correlate perceived grittiness to the size, shape, and identity of surface-situated crystals.

\section{MATERIALS AND METHODS}

\section{Cheese Samples}

Commercial soft smear-ripened cheeses $(\mathrm{n}=24)$ were sourced from the United States and Europe (France, Italy, Spain, and Germany). Replicate wheels of cheese with the same code date and vat identifier (if available) were used for all analyses. All samples were purchased from retail establishments or were shipped overnight on ice and inspected upon arrival. Samples were stored at $3^{\circ} \mathrm{C}$ for up to $5 \mathrm{~d}$ until analyses were conducted. Each of the cheeses used in our study is referred to with a single letter (A through X).

Surface $\mathrm{pH}$ measurements were taken on 3 separate locations (bottom, side, and top) on each wheel, using a flat-tipped probe (Thomas Scientific, Swedesboro, NJ), and averaged for each cheese ( 6 total $\mathrm{pH}$ measurements per cheese). Moisture content was determined in triplicate using a forced-air draft oven set to $100^{\circ} \mathrm{C}$. A wedge from each wheel was ground to homogeneity using a mortar and pestle. Approximately $2 \mathrm{~g}$ of homogenized sample were weighed and transferred into predried and preweighed aluminum pans. Samples were dried for 24 h (6 total moisture measurements per cheese).

\section{Cheese Smear Collection and Processing}

Three $1-\mathrm{cm}^{2}$ rind sections of smear material from each wheel were collected from the top, bottom, and side wheel surfaces using a metal spatula. Gentle pressure was used to limit the possibility of crystals being fractured. For PXRD analysis, alkaline dispersions $(\mathrm{pH}$ $=10$ ) of smear samples were used. Dispersions were prepared by placing the smear sample in a $50-\mathrm{mL}$ beaker and covering with $15 \mathrm{~mL}$ of $0.0001 \mathrm{M} \mathrm{NaOH}$ (Fisher Scientific, Pittsburgh, PA), which were then vortexed gently to encourage good dispersion and allowed to rest at room temperature for approximately $2 \mathrm{~h}$. Crystals that settled to the bottom of the beaker during this time were used for PXRD analysis. Alkaline dispersion was used to lower the amount of amorphous material present in the cheese smears, thereby lowering the background noise in the diffractograms and improving PXRD performance overall. For PLM analysis, smear samples were applied directly to glass microscope slides. Alkaline dispersion was not used for PLM analysis, because our previous work indicated that crystal size may be reduced when an alkaline dispersion methodology is used to harvest crystals, perhaps due to dissolution of the crystals in the aqueous environment (Polowsky et al., 2018).

\section{PXRD}

A $300-\mu \mathrm{L}$ aliquot of alkaline smear dispersion liquid (pipetted from bottom of beaker) was applied to a zerobackground diffraction slide for all PXRD analyses. Care was taken to ensure the dispersion liquid was applied to the center of the slide to allow for equal distribution of crystals on slide. Excess liquid was absorbed 
by blotting with a tissue (Kimwipes, Kimberly-Clark Professional, Roswell, GA). Diffraction patterns were generated using a Miniflex II powder X-ray diffractometer (Rigaku, The Woodlands, TX). Patterns were created between $5^{\circ} 2 \theta$ and $50^{\circ} 2 \theta$ (20 represents the angle formed between the X-ray source, diffraction slide, and X-ray detector), using a speed of $2^{\circ} 2 \theta / \mathrm{min}$. The PDXL software package (Rigaku) was used to analyze the diffractograms. Each experimental pattern was compared with existing reference diffraction patterns in the International Center for Diffraction Data database (http: //www.icdd.com). The following International Center for Diffraction Data reference cards were used: 01-0747147 (ikaite), 01-074-3640 (brushite), 00-005-0586 (calcite), 00-015-0762 (struvite), and 00-039-1840 (L-tyrosine). Peaks from experimental patterns were compared with reference patterns until all peaks were identified. Preferred orientation was present in several of the experimental diffraction patterns but did not inhibit identification of major crystalline phases, as demonstrated previously (Polowsky et al., 2018).

\section{Polarized Light Microscopy}

Smear scrapings applied to a glass microscope slide (as described above) were overlaid with a thin film of mineral oil to delay crystal dehydration. Micrographs of smear scrapings were captured using a Nikon E200POL microscope (Nikon Corporation, Tokyo, Japan) equipped with a rotating stage. Samples were viewed under polarized light (crossed polarizers) using a $1 / 4 \lambda$ plate (quartz filter), and images were captured using a SPOT Idea 1.3 MP color camera (SPOT Imaging Solutions, Sterling Heights, MI). Presumptive entities were confirmed to be crystals when they displayed uniform extinction upon rotating the stage a full $360^{\circ}$. Four fields of view (FOV) were captured for each cheese wheel duplicate. The FOV were chosen at random using a systematic random sampling method (Altunkaynak et al., 2011). Gridded microscope slide stickers (Sigma-Aldrich, St. Louis, MO) were applied to back side of each microscope slide. Grid squares were chosen at random and centered in each FOV before an image was captured (8 images total were captured per cheese).

\section{Image Analysis}

Images from PLM were imported into Adobe Photoshop (Adobe Systems Inc., San Jose, CA), where a black background was applied and crystals were outlined using the quick selection tool. Crystals that were overlapping, crystals obscured by smear material, and crystals not entirely in the FOV were not used for any further analysis. Outlined images were saved as PNG files and analyzed using Fiji (ImageJ distribution; Schindelin et al., 2012). Each image was converted to black and white (8-bit images), and thresholding was applied using the Li's Minimum Cross Entropy thresholding option ( $\mathrm{Li}$ and Lee, 1993). Each image was then analyzed using the particle analysis plugin. Area, perimeter, and circularity measurements were collected and recorded for each isolated crystal in each processed image. Circularity, in ImageJ, is calculated using the following formula: $4 \pi\left(\right.$ area/perimeter $\left.{ }^{2}\right)$, where circularity of 1.00 indicates a perfect circle, and circularity of 0.00 indicates an increasingly extended polygon. A minimum size threshold of 50 pixels $^{2}$ was used; objects smaller than this could not be accurately measured and were not used in any further analysis.

\section{Descriptive Sensory Analysis}

A trained sensory panel $(\mathrm{n}=12)$ evaluated all samples in duplicate using the Spectrum method (Meilgaard et al., 2007). A 15-point intensity scale was used for each attribute. Sensory panelists were recruited from the staff of The Cellars at Japer Hill (Greensboro, VT), and had extensive prior experience evaluating smearripened cheeses. Each panelist underwent an additional $15 \mathrm{~h}$ of training specific to our study. Attribute definitions, evaluation techniques, and attribute references can be seen in Table 1.

A 13-mm cross section of each cheese, consisting of 2 pieces of rind separated by the cheese body, were stored in small soufflé cups labeled with random 3-digit codes. All samples were evaluated at a temperature of $15^{\circ} \mathrm{C}$. Panelists expectorated all samples and were provided with deionized water and unsalted crackers as palate cleansers. RedJade software (RedJade Software Solutions LLC, San Francisco, CA) was used to organize descriptive sensory data and to track panelist performance. Grittiness threshold was determined in a manner similar to that as described by Imai et al. (1995), based on the number of panelists that perceived no grittiness. All sensory analysis was performed in compliance of University of Vermont's Institutional Review Board guidelines for human subjects.

\section{Statistical Analysis}

All statistical analyses were performed using $\mathrm{R}$ (version 3.3.2; R Core Team, 2016). Compositional data, crystal size and shape data, and descriptive sensory data were analyzed via one-way ANOVA (using the aov function), with Tukey's Honest Significance Difference (HSD) test used to check for significant differences between means $(P<0.05)$. The "agricolae" package was 
Table 1. Attribute lexicon used for descriptive sensory analysis

\begin{tabular}{|c|c|c|c|}
\hline Attribute & Definition & Technique & References (0-15 scale) \\
\hline Acid & $\begin{array}{l}\text { Basic taste elicited by lactic } \\
\text { acid }\end{array}$ & Assess the taste of the cheese body & $\begin{array}{l}0=\text { water; } \\
4=0.075 \% \text { lactic } \text { acid }^{7} \text { solution } \\
8=0.100 \% \text { lactic acid solution }\end{array}$ \\
\hline
\end{tabular}

\footnotetext{
${ }^{1}$ Hannaford Ammonia Clear; Delhaize Group, Brussels, Belgium.

${ }^{2}$ Philadelphia Cream Cheese; Kraft Heinz, Chicago, IL.

${ }^{3}$ Crisco Vegetable Shortening; J.M. Smucker Company, Orrville, OH.

${ }^{4}$ Domino Superfine Sugar; American Sugar Refining Inc., West Palm Beach, FL.

${ }^{5}$ Domino Granulated Sugar; American Sugar Refining Inc.

${ }^{6}$ Caffeine Powder (USP/FCC); Fisher Scientific, Hampton, NH.

${ }^{7}$ Lactic Acid (88\% Liquid); Nelson-Jameson, Marshfield, WI.

${ }^{8}$ Morton Salt (uniodized); Morton Salt, Chicago, IL.
}

used for the Tukey HSD test (version 1.2-8; de Mendiburu, 2017). Principal component analysis (PCA) was also applied to these data using the "FactoMineR" package (version 1.34; Lê et al., 2008); PCA was carried out using the correlation matrix. Pearson correlation coefficients were generated using the "cor" function. Grittiness occurrence and PXRD data were analyzed via a chi-squared test using the "vcd" package (version 1.4-4; Meyer et al., 2017).

\section{RESULTS}

\section{Cheese Samples}

Place of origin, moisture content, and $\mathrm{pH}$ values of the cheeses used in our study are shown in Table 2. Moisture content ranged from 40.2 to $54.5 \%$, with a mean value of $48.4 \%$. Twenty-two cheeses $(92 \%)$ had moisture contents between 45 and $60 \%$ and were classified as semi-soft smear-ripened cheeses (Bockelmann, 2011). Two cheeses fell below this moisture range. Surface $\mathrm{pH}$ ranged from approximately 6.63 to 8.03 ; high surface $\mathrm{pH}$ is typical of smear-ripened cheeses due to extensive surface deacidification during ripening (Bockelmann, 2011). These differences could have resulted from differences in make schedule, affinage practices, or the age at which the cheeses were examined.

\section{PXRD}

A representative diffractogram obtained from cheese E can be seen in Figure 1, which highlights the crystal peaks identified in a single cheese. Results from PXRD crystal identification are summarized in Table 3. Crystals were detected in 23 out of the $24(\sim 96 \%)$ cheeses, with only 1 cheese (cheese P) showing no identifiable peaks present in its diffractogram. This could be due to large amounts of amorphous material obscuring crystalline entities, as previously observed (Polowsky et al., 2018). Overall, we identified struvite, ikaite, brushite, calcite, and the AA tyrosine in cheeses. Of the samples with crystals present, struvite, ikaite, brushite, calcite, and tyrosine had approximate rates of occurrence of $100,70,26,17$, and $4 \%$, respectively. Worthy of mention is the occurrence of multiple crystal types in a single cheese, which the majority (17 out of 24 ) of samples exhibited.

\section{PLM and Image Analysis}

The results of crystal identification via PLM and crystal metrics resulting from image analysis of PLM micrographs are shown in Table 3. Identification via PLM of ikaite and struvite crystals was carried out using the criteria described in our previous work 
Table 2. Production location and surface $\mathrm{pH}$ and moisture determinations of cheeses surveyed in this study ${ }^{1}$

\begin{tabular}{|c|c|c|c|c|c|}
\hline \multirow[b]{2}{*}{ Cheese } & \multirow[b]{2}{*}{ Production location } & \multicolumn{2}{|c|}{ Surface $\mathrm{pH}$} & \multicolumn{2}{|c|}{ Moisture content (\%) } \\
\hline & & Mean & SD & Mean & $\mathrm{SD}$ \\
\hline A & Vermont & 7.23 & 0.16 & 47.6 & 0.39 \\
\hline B & New York & 8.03 & 0.19 & 44.8 & 0.80 \\
\hline $\mathrm{C}$ & Virginia & 7.28 & 0.02 & 48.0 & 0.69 \\
\hline $\mathrm{D}$ & Vermont & 7.91 & 0.11 & 51.3 & 0.13 \\
\hline $\mathrm{E}$ & Vermont & 7.70 & 0.12 & 53.8 & 0.30 \\
\hline $\mathrm{F}$ & Vermont & 6.96 & 0.07 & 47.9 & 0.89 \\
\hline G & California & 6.49 & 0.09 & 46.9 & 0.49 \\
\hline $\mathrm{H}$ & Wisconsin & 7.18 & 0.09 & 45.9 & 0.76 \\
\hline I & Minnesota & 7.67 & 0.06 & 47.3 & 0.11 \\
\hline$J$ & Wisconsin & 7.53 & 0.03 & 49.1 & 0.83 \\
\hline K & California & 7.45 & 0.05 & 47.8 & 0.14 \\
\hline $\mathrm{L}$ & Indiana & 7.66 & 0.08 & 52.6 & 0.13 \\
\hline M & New York & 7.98 & 0.09 & 49.7 & 0.20 \\
\hline $\mathrm{N}$ & Colorado & 7.50 & 0.01 & 50.4 & 0.35 \\
\hline $\mathrm{O}$ & Colorado & 7.58 & 0.04 & 40.2 & 0.14 \\
\hline $\mathrm{P}$ & Indiana & 7.67 & 0.16 & 53.2 & 0.35 \\
\hline Q & France & 7.19 & 0.08 & 48.6 & 0.08 \\
\hline $\mathrm{R}$ & France & 7.19 & 0.01 & 54.5 & 0.20 \\
\hline S & Italy & 7.10 & 0.03 & 50.5 & 0.37 \\
\hline $\mathrm{T}$ & Italy & 7.32 & 0.05 & 52.9 & 0.78 \\
\hline $\mathrm{U}$ & France & 7.12 & 0.04 & 49.4 & 0.12 \\
\hline $\mathrm{V}$ & France & 6.63 & 0.11 & 46.3 & 0.08 \\
\hline $\mathrm{W}$ & Spain & 6.82 & 0.05 & 53.2 & 0.09 \\
\hline $\mathrm{X}$ & Germany & 6.64 & 0.04 & 48.1 & 0.19 \\
\hline $\mathrm{HSD}^{2}$ & & 0.36 & & 1.84 & \\
\hline
\end{tabular}

${ }^{1}$ Means and SD are results from triplicate measurements from duplicate wheels of cheese.

${ }^{2}$ Means that differ by honestly significant difference (HSD) within each column are significantly different $(P$ $<0.05)$.

(Polowsky et al., 2018), namely birefringence and angle of extinction. Such criteria have not been developed for the other crystal types present in our study. However, please note that crystal metrics (length, area, and circularity) were determined from all suitable single crystal entities regardless of their identity standing. Overall, incomplete agreement was noted between PLM identification and PXRD identification. In 7 instances (cheeses B, G, H, L, Q, V, and W) did PLM analysis fail to detect ikaite or struvite when those crystal types were detected by PXRD. Of special interest are cheeses $\mathrm{S}$ and T, where PLM identified struvite or ikaite but PXRD analysis did not. As described in our previous work (Polowsky et al., 2018), amorphous smear material can obscure crystals during PLM analysis, making accurate identification difficult. The alkaline dispersion sample preparation method used for PXRD analysis removed much of this amorphous background, leading to more accurate crystal identification. Future work is needed to improve the fidelity of crystal identification via PLM, as well as criteria for identifying calcite and brushite via PLM.

Crystal length and crystal area varied by 2 to 3 orders of magnitude, respectively. Crystal length ranged from 27 to 1,096, $\mu \mathrm{m}$, with an overall average value of approximately $235 \mu \mathrm{m}$. Crystal area ranged from approximately 513 to $214,000 \mu^{2}$, with an overall average value of approximately $44,000 \mu^{2}$. Crystal circularity ranged from approximately 0.481 to 0.958 , with an overall average value of approximately 0.760 . Figure 2 shows an example of PLM micrographs that were used for presumptive crystal identification and image analysis; intra- and intersample differences in crystal shape, crystal size, and coloring are clearly visible. These results indicate a diverse collection of crystal sizes and morphologies existed on the surfaces of the soft washed-rind cheeses used in our study. Attempts were made to accurately quantify crystal quantity in a manner similar to that of Hough et al. (1990). Crystal breakage and inconsistent distribution of crystals on the cheese rinds did not allow for accurate measurements. Further work is needed to develop a robust method for quantifying number of crystals present on the surface of smear-ripened cheeses.

\section{Descriptive Sensory Analysis}

Table 4 contains the results from the descriptive sensory analysis. All attributes, except grittiness, were detected in each of the cheeses evaluated. Mean grittiness scores varied widely depending on cheese, with a range from approximately 0.0 to 7.5 . Grittiness, when 


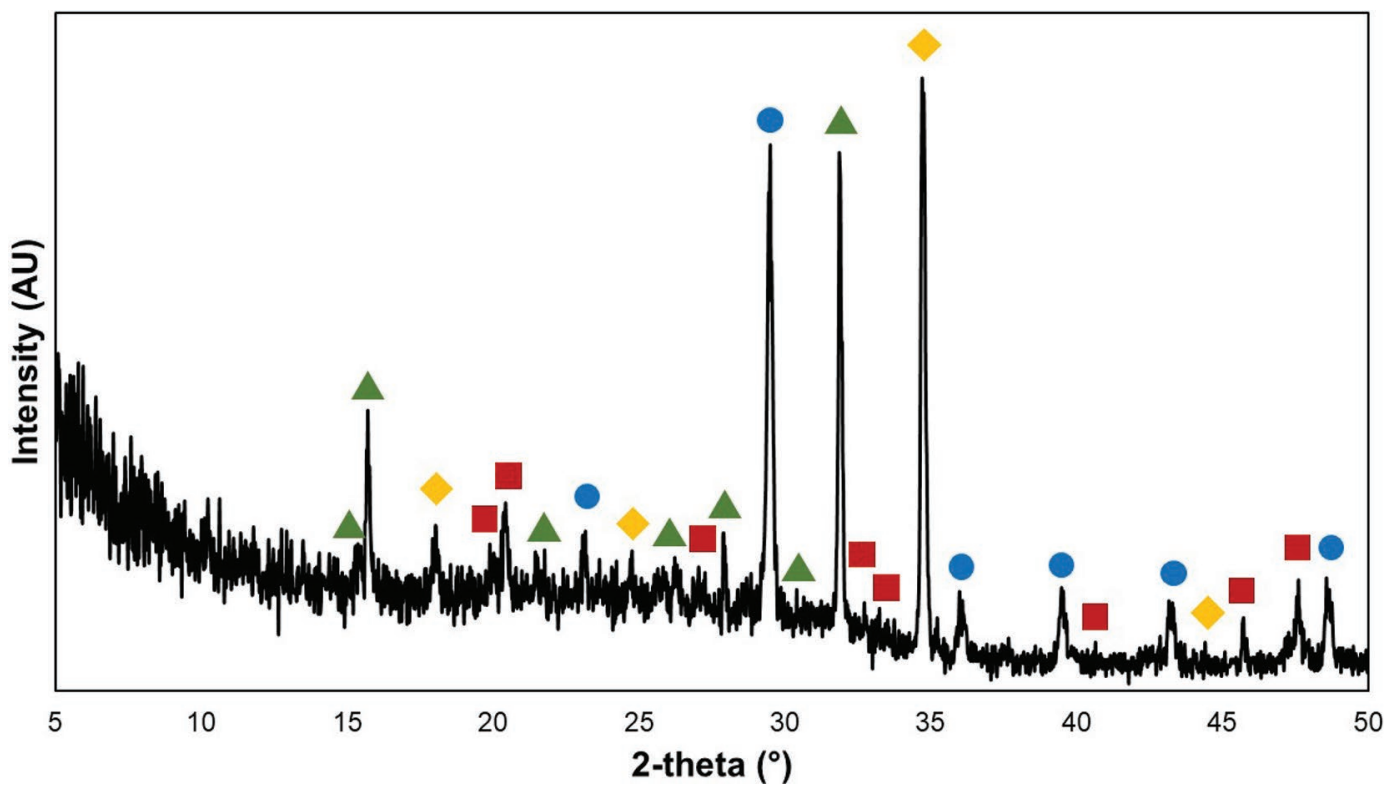

Figure 1. An example powder X-ray diffractometry (PXRD) pattern captured from crystals isolated from cheese C. Characteristic peaks of ikaite, struvite, calcite, and brushite are marked with squares, triangles, circles, and diamonds, respectively. AU = arbitrary units. Color version available online.

Table 3. Summary of polarized light microscopy (PLM) data (crystal length, crystal area, circularity) and powder X-ray diffractometry (PXRD) observations (crystals types)

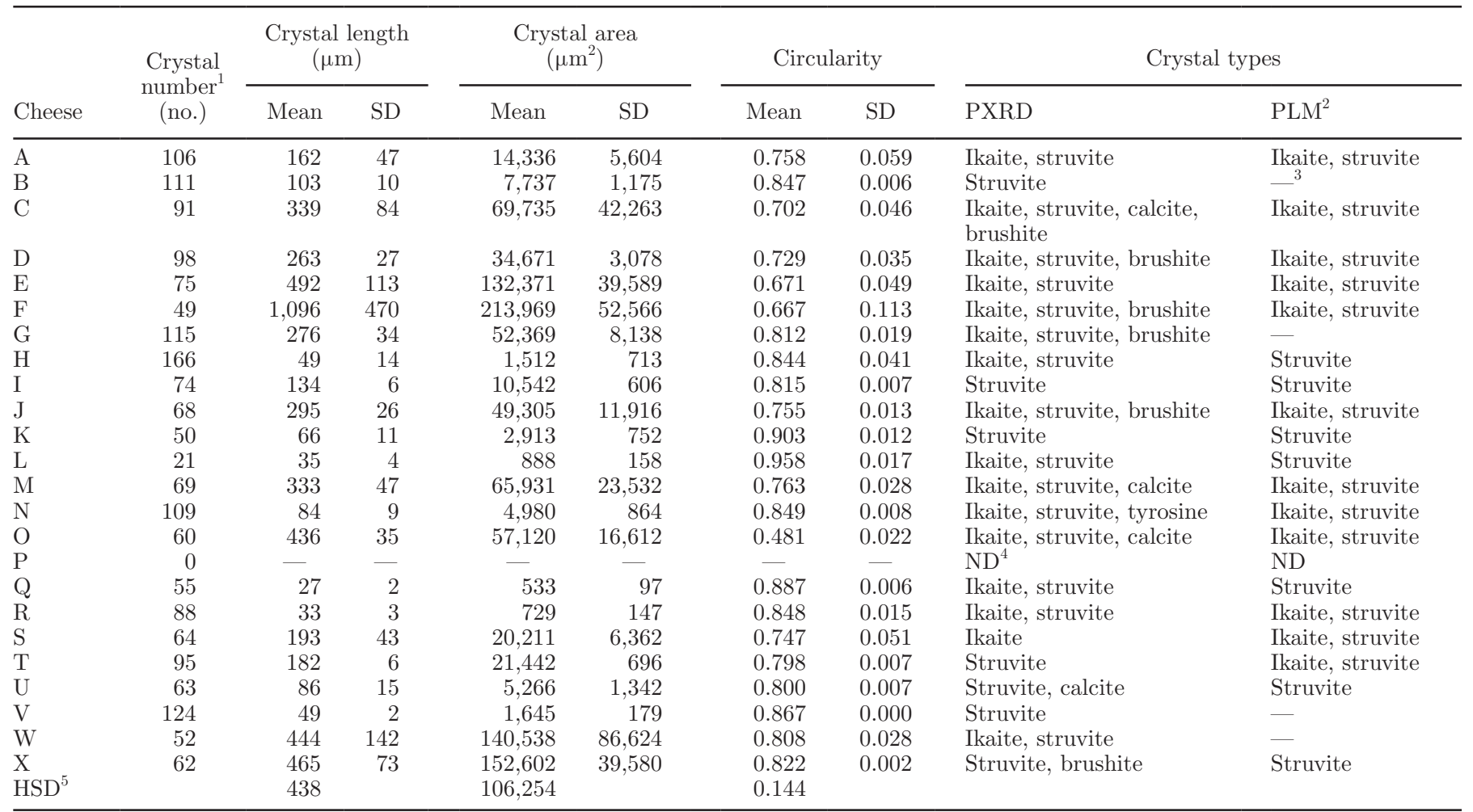

${ }^{1}$ Number of crystals used for PLM analysis: determination of crystal length, crystal area, and circularity.

${ }^{2}$ Crystals identified via polarized light microscopy methodology as described in Polowsky et al. (2018).

${ }^{3}$ Crystals were detected, but no conclusive identification could be made.

${ }^{4}$ No crystals detected.

${ }^{5}$ Means that differ by honestly significant difference (HSD) within each column are significantly different $(P<0.05)$. 
Table 4. Summary of descriptive sensory analysis data (means and SD of panel duplicates)

\begin{tabular}{|c|c|c|c|c|c|c|c|c|c|c|}
\hline \multirow[b]{2}{*}{ Cheese } & \multicolumn{2}{|c|}{ Ammonia aroma } & \multicolumn{2}{|c|}{ Grittiness } & \multicolumn{2}{|c|}{ Bitter } & \multicolumn{2}{|c|}{ Acid } & \multicolumn{2}{|c|}{ Salt } \\
\hline & Mean & $\mathrm{SD}$ & Mean & $\mathrm{SD}$ & Mean & $\mathrm{SD}$ & Mean & $\mathrm{SD}$ & Mean & $\mathrm{SD}$ \\
\hline $\bar{A}$ & 3.9 & 0.1 & 2.6 & 0.2 & 4.4 & 9.1 & 0.4 & 3.7 & 0.4 & 4.2 \\
\hline B & 8.5 & 0.2 & 1.6 & 1.3 & 1.1 & 6.4 & 1.4 & 2.9 & 0.5 & 5.6 \\
\hline $\mathrm{C}$ & 2.9 & 0.1 & 3.4 & 0.6 & 3.6 & 5.9 & 1.8 & 3.8 & 0.8 & 3.9 \\
\hline $\mathrm{D}$ & 7.5 & 0.5 & 3.1 & 1.1 & 5.7 & 9.0 & 1.1 & 4.1 & 0.5 & 5.8 \\
\hline $\mathrm{E}$ & 6.7 & 0.2 & 3.9 & 0.6 & 6.3 & 5.7 & 0.3 & 3.8 & 0.0 & 5.9 \\
\hline $\mathrm{F}$ & 3.1 & 1.0 & 7.4 & 0.4 & 4.4 & 6.0 & 0.5 & 3.2 & 0.2 & 3.9 \\
\hline G & 2.4 & 0.5 & 2.8 & 0.5 & 1.7 & 3.9 & 0.1 & 3.0 & 0.5 & 5.3 \\
\hline $\mathrm{H}^{1}$ & 3.8 & 0.4 & 0.1 & 0.0 & 0.1 & 3.4 & 0.1 & 2.4 & 0.3 & 4.4 \\
\hline $\mathrm{I}$ & 4.9 & 0.2 & 1.8 & 0.8 & 1.5 & 8.2 & 0.5 & 4.2 & 0.5 & 3.8 \\
\hline $\mathrm{J}$ & 7.4 & 0.2 & 3.7 & 1.6 & 3.4 & 6.6 & 0.6 & 5.2 & 1.4 & 6.7 \\
\hline $\mathrm{K}^{1}$ & 4.8 & 0.1 & 0.1 & 0.0 & 0.9 & 3.5 & 0.2 & 4.0 & 0.3 & 4.7 \\
\hline $\mathrm{L}^{1}$ & 4.6 & 0.2 & 0.2 & 0.2 & 0.4 & 2.6 & 0.2 & 5.2 & 0.5 & 7.0 \\
\hline $\mathrm{M}$ & 7.3 & 0.1 & 2.7 & 0.5 & 3.9 & 5.1 & 0.5 & 4.4 & 0.1 & 6.4 \\
\hline $\mathrm{N}$ & 4.6 & 0.2 & 1.9 & 0.3 & 3.4 & 3.6 & 0.8 & 5.7 & 0.2 & 6.6 \\
\hline $\mathrm{O}$ & 4.6 & 0.5 & 3.6 & 0.5 & 4.7 & 2.6 & 0.4 & 5.4 & 0.1 & 6.7 \\
\hline $\mathrm{P}^{1}$ & 7.4 & 0.8 & 0.0 & 0.0 & 0.0 & 7.0 & 0.9 & 5.1 & 0.9 & 5.9 \\
\hline $\mathrm{Q}^{1}$ & 5.5 & 0.0 & 0.0 & 0.0 & 0.0 & 4.0 & 1.9 & 4.7 & 0.5 & 6.2 \\
\hline $\mathrm{R}^{1}$ & 6.7 & 0.2 & 0.4 & 0.6 & 0.2 & 9.2 & 0.2 & 3.0 & 0.2 & 6.5 \\
\hline $\mathrm{S}$ & 2.3 & 0.6 & 2.1 & 0.2 & 4.8 & 1.4 & 0.2 & 3.2 & 0.2 & 3.7 \\
\hline $\mathrm{T}$ & 4.0 & 0.0 & 2.0 & 1.2 & 6.3 & 2.3 & 0.7 & 3.8 & 0.1 & 5.5 \\
\hline $\mathrm{U}$ & 5.8 & 1.8 & 1.6 & 0.4 & 3.4 & 7.8 & 0.5 & 2.9 & 0.6 & 3.7 \\
\hline $\mathrm{V}^{1}$ & 1.1 & 0.1 & 0.2 & 0.0 & 0.2 & 1.5 & 0.8 & 4.6 & 1.3 & 2.7 \\
\hline W & 4.9 & 0.4 & 5.5 & 0.6 & 4.6 & 3.6 & 0.1 & 4.7 & 0.9 & 5.7 \\
\hline $\mathrm{X}$ & 1.6 & 0.1 & 5.3 & 1.2 & 2.1 & 3.7 & 0.2 & 4.9 & 0.4 & 4.7 \\
\hline $\mathrm{HSD}^{2}$ & 2.12 & & 2.87 & & 3.16 & & 2.43 & & 1.49 & \\
\hline
\end{tabular}

${ }^{1}$ Cheeses with grittiness intensity below sensory threshold (i.e., at least $75 \%$ of panelists indicated 0 for grittiness intensity).

${ }^{2}$ Means that differ by honestly significant difference (HSD) within each column are significantly different $(P<0.05)$.

detected, was on the rinds of the cheeses used in our study, with panelists indicating no perceived grittiness in the cheese body or paste. Panelists determined that 2 of the cheeses ( $\mathrm{P}$ and $\mathrm{Q}$ ) contained no perceptible grittiness and had mean values of 0 for grittiness. Five other cheeses (H, K, L, R, and V) had very low grittiness scores (below 0.5), with most the panelists indicating 0 for grittiness. The sensory threshold of grittiness was established in a manner similar to that described by Imai et al., (1995). Cheeses where at least $75 \%$ of pan- elists perceived zero grittiness in both replicates were considered below the threshold and therefore not gritty. Mean scores for ammonia aroma, bitterness, and salt also varied somewhat widely, with approximate ranges of 1.1 to $8.5,1.4$ to 9.2 , and 2.7 to 7.0 , respectively.

Principal component analysis of sensory data, cheese compositional data, and crystal size and shape data was performed (Figure 3). Principal component (PC) 1 accounted for approximately $32 \%$ of the variation in the data and was composed mainly of crystal area,

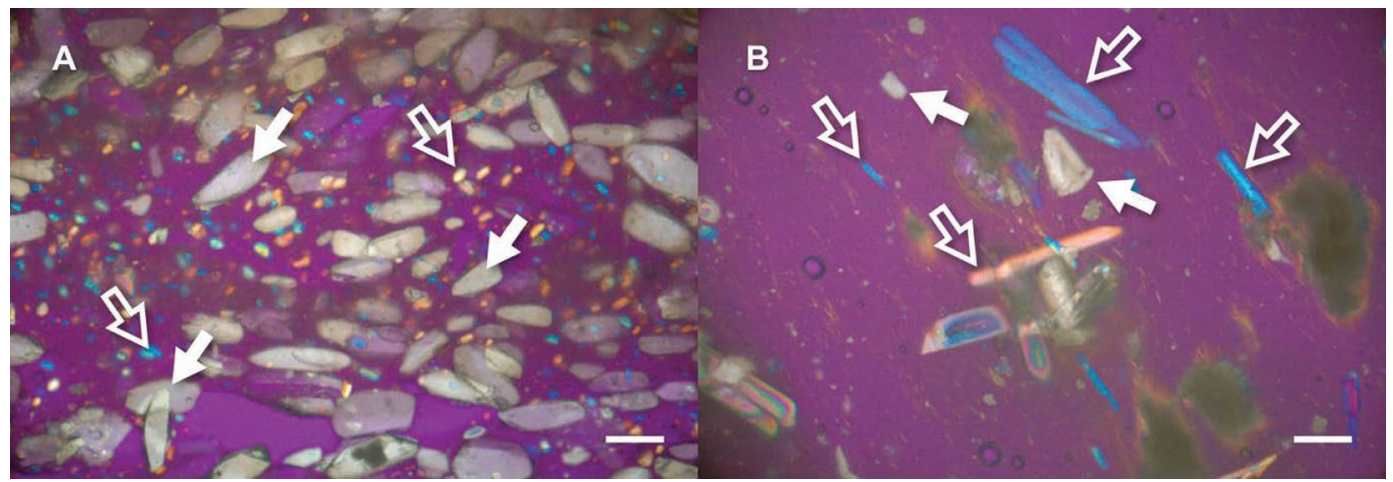

Figure 2. Polarized light micrographs of crystals isolated from cheese smear material. Crystals of ikaite (solid arrows) and struvite (hollow arrows) are shown. Panel A contains crystals high in circularity (rounder crystals), whereas panel B contains crystals lower in circularity (more boxy or needle-like crystals). Scale bars represent $250 \mu \mathrm{m}$. Color version available online. 


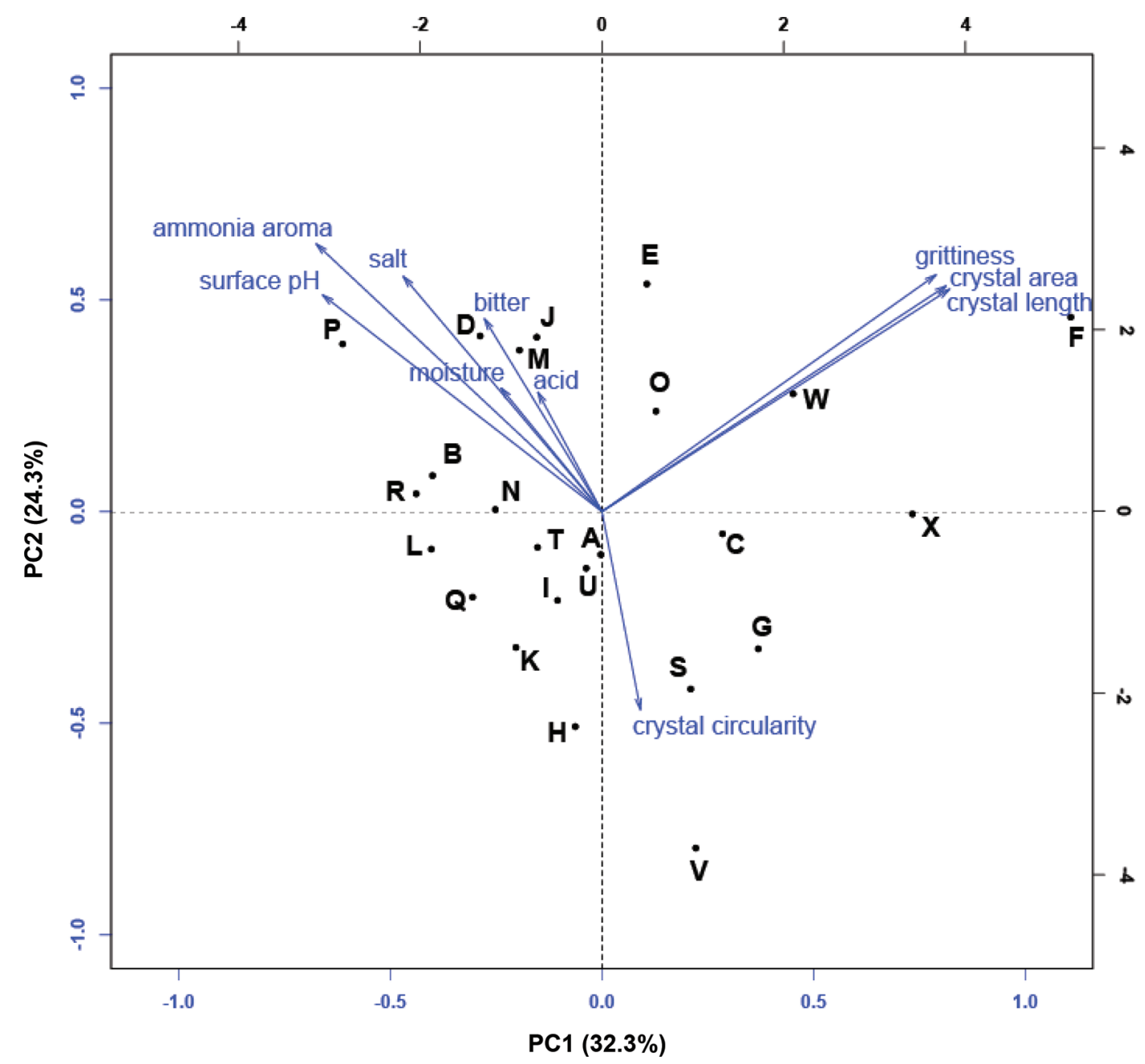

Figure 3. Principal component (PC) analysis biplot of cheese compositional data, sensory data, and crystal size and shape metrics. Letters represent cheese samples. Principle components 1 and 2 are shown. Color version available online.

crystal length, and grittiness. Principal component 2 explained approximately $24 \%$ of the variation, and was composed mainly of ammonia aroma, grittiness, and salt. Principal components 3 and 4 were composed of acid, bitter, salt, moisture, and crystal circularity (data not shown). Approximately $25 \%$ of the variation in the data was explained by PC 3 and 4 (data not shown).

\section{DISCUSSION}

\section{Significance of Crystal Types Identified}

Ikaite, calcite, struvite, and brushite were found on multiple cheeses used in our study. Each of these crystal types have been identified previously on soft smearripened cheese (Tansman et al., 2017a,c). Previous studies have included only a few cheeses, largely from 1 producer. Our study incorporated 24 cheeses from 17 different producers located in the United States and Europe. The results of the present work indicated that these crystals are common among soft smear-ripened cheeses, and are not unique to a certain producer or region. Instead, these crystals are the result of physicoor biochemical reactions common in this cheese variety, namely surface microbe metabolism and mineral migration.

A few consequences of this microbe metabolism include deacidification of the cheese surface and subsequent mineral precipitation, as well as proteolysis (McSweeney, 2004). Surface precipitation and crystallization establishes concentration gradients, which encourages radial migration of mineral elements, such as calcium, magnesium, and phosphorus (Tansman et al., 2017a). Gaseous products, including carbon dioxide and ammonia, are also formed during ripening of washed-rind cheeses (Gori et al., 2007; Hélias et al., 
2007). These surface-situated minerals (i.e., Ca, Mg, and $\left.\mathrm{PO}_{4}\right)$ and gaseous products $\left(\mathrm{CO}_{2}\right.$ and $\left.\mathrm{NH}_{3}\right)$ are the requisite components of the lattice structures of the various observed crystals. Large concentrations of these components accumulate on the cheese surface and most likely surpass the solubility threshold and form precipitates and crystals.

The pronounced occurrence of struvite is notable, as every sample that contained crystals contained struvite. Struvite has been reported in other biological systems, notably wastewater processing (Kim et al., 2016), urinary stones (Wilsenach et al., 2007), and fish products (Amano, 1950). Although a main component of struvite, magnesium occurs in cheese at much lower amounts than calcium, these crystals were omnipresent in the samples used in our study. Evidently, the conditions that occur at the surface of smear-ripened cheeses strongly favor struvite crystallization. These conditions include high phosphate content (Tansman et al., 2017a), high pH (see Table 2), and presumably high dissolved ammonia content because smear organisms are generally proteolytic and release large amounts of ammonia into the atmosphere via deamination of AA (Brennan et al., 2004). Struvite has a very low solubility around pH 7 (Kim et al., 2016), and crystallization is very sensitive to increased phosphate concentration (Wu et al., 2005), which may have contributed to the high incidence of struvite in present study despite low magnesium levels relative to calcium in cheese in general.

Calcite and its metastable form ikaite were present in many of the samples. These crystals are both of the type calcium carbonate, which are also sparingly soluble at the neutral or high $\mathrm{pH}$ range. As mentioned, the surface accumulation of calcium has been well established in surface-ripened cheeses (Tansman et al., 2017a,b). Carbon dioxide content of cheese ripening spaces can reach high levels (Picque et al., 2006), which can dissolve into the water phase at the cheese surface. At high enough concentrations, these components exceed solubility thresholds and crystallize. Of note is the formation of metastable ikaite, which has rarely been seen in nature (Rysgaard et al., 2012). Ikaite and calcite are known to transform into each other depending on reaction conditions, and the presence of phosphate can stabilize metastable ikaite (Hu et al., 2014). In previous work, although surface crystals of calcite persisted in smear-ripened cheeses, crystals of ikaite and struvite became the prominent species as ripening progressed, as evidenced in PLM (Tansman et al., 2017a; Polowsky et al., 2018). It is possible that the surface smear of smear-ripened cheeses present an optimal environment for ikaite to form and for calcite to transform into ikaite, which may account for the absence of calcite in some cheeses. More research is needed to determine if that is the case.

Brushite crystals are likely the easiest to account for on the surface of smear-ripened cheeses. Calcium and phosphate are major structural components of the casein matrix (Lucey and Fox, 1993) and undergo the aforementioned radial migration. Brushite is also insoluble in the $\mathrm{pH}$ range of many washed-rind cheeses (Arifuzzaman and Rohani, 2004). Although brushite is a metastable form of calcium phosphate, certain chemical kinetic conditions can encourage its formation, such as concentration of ions and $\mathrm{pH}$ (Arifuzzaman and Rohani, 2004). It is possible that brushite dissolved as ikaite and struvite crystallized during ripening. More research is needed to determine if this was the case.

Tyrosine crystals were found on only 1 sample (cheese N). Although this crystal type has not been identified previously on soft washed-rind cheese, it commonly occurs in Grana-style cheeses (Tansman et al., 2015). A consequence of the surface microbiota's proteolytic behavior could be the accumulation of free AA (Brennan et al., 2002), such as tyrosine. High concentration of free tyrosine, coupled with its low solubility at neutral or alkaline $\mathrm{pH}$ (Hitchcock, 1924), may explain its presence. However, it is unclear why tyrosine was only found on 1 sample. A possible explanation may be the specific microbial metabolism of cheese $\mathrm{N}$ resulted in unusually high amounts of free tyrosine. However, this needs to be confirmed through future research because free tyrosine was not measured in the present study.

\section{Grittiness Threshold and Descriptive Sensory Analysis}

To our knowledge, surface grittiness in washed-rind cheese has only been mentioned once in the dairy technology literature (Le Mens et al., 2000), but never associated with crystal growth. Although the occurrence of this attribute has not been thoroughly confirmed, it is a recognized attribute in the cheese industry. To accurately measure grittiness as a descriptive sensory attribute, a novel method was needed to establish reference samples for grittiness. Mixtures of vegetable shortening with different grain sizes of sugar were used in the present work as anchor points (Table 1). Vegetable shortening was chosen rather than other dairy-containing matrices (i.e., cream cheese) due to its low moisture content, which ensured crystals did not dissolve over time. Sugar was chosen as the crystalline inclusion due to its mild sweet taste, which would not interfere with other common taste attributes associated with washed-rind cheeses (e.g., salt and bitter). The de- 


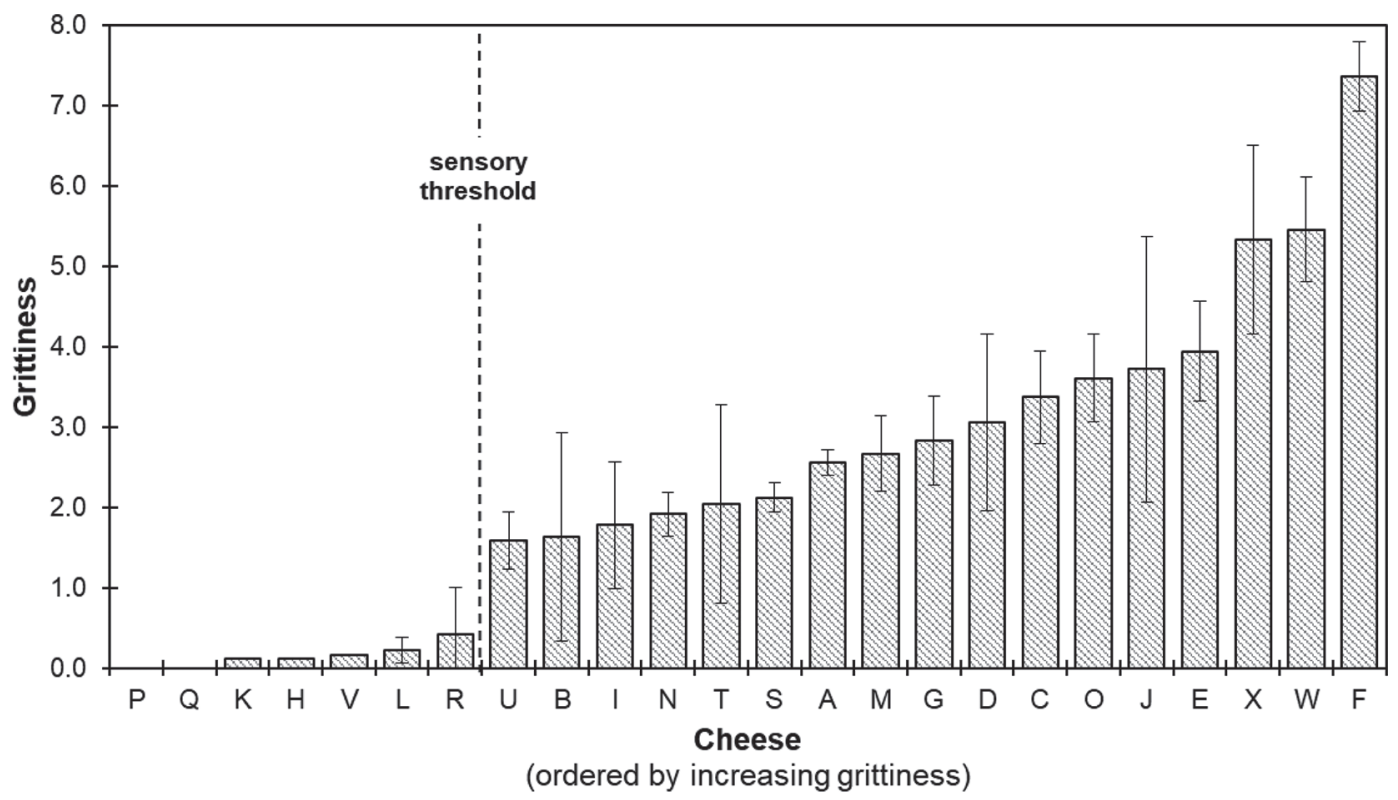

Figure 4. Mean grittiness scores for cheeses used in this study. Error bars show 1 SD from the mean. Cheeses with grittiness value below sensory threshold had at least $75 \%$ of panelists indicate 0 for grittiness.

scriptive panel agreed that these reference samples allowed for consistent grittiness evaluation, with minimal chance of carryover effects when used in conjunction with evaluating cheese samples.

Perceived grittiness varied by a large amount across the 24 samples evaluated in our study (Figure 4), from no grittiness to easily identifiable grittiness. Seven cheeses met the criteria for the absence of grittiness (cheeses $\mathrm{P}$, $\mathrm{Q}, \mathrm{K}, \mathrm{H}, \mathrm{V}, \mathrm{L}$, and R). Coincidently, these 7 cheeses had mean grittiness values below 0.5 , whereas cheeses above the threshold had grittiness values of at least 1.5. Figure 4 indicates this threshold with a dashed line. In the present study, $71 \%$ of cheeses were considered gritty. Panelists' comments indicated that grittiness of approximately 7.5 (cheese F) elicited a sensation similar to that of chewing sand between the molars. This relatively high occurrence of grittiness confirms what anecdotal evidence suggests; that is, grittiness is a common sensorial attribute associated with soft washedrind cheeses. The PCA of crystal metrics and grittiness data (Figure 3) indicated a strong association between crystal size and grittiness. Comparing the occurrence of grittiness to mean crystal size allows for an approximate estimate of the threshold size of crystals, above which grittiness could occur. Figure 5 shows the mean size distribution (length and area) of crystals across all 24 cheese samples. The grittiness threshold is indicated with a dashed line, which occurred at approximately at 66 and $2,900 \mathrm{\mu m}^{2}$. The grittiness threshold is indicated with a dashed line in Figure 5. The 7 cheeses that fell below the sensory threshold for grittiness also had the lowest mean crystal lengths and areas of the 24 surveyed cheeses. These crystal sizes serve as approximate size thresholds, and mean crystal values at or above this level would likely result in gritty cheese. It is important to note that results from a descriptive panel do not necessarily equate to how consumers perceive grittiness. Further studies are needed to determine at what crystal size consumers perceive grittiness, and at what grittiness intensity are cheeses considered objectionable. Grittiness was not correlated with any of the other sensory attributes measured in this study (data not shown).

Other trends of interest include the association between ammonia aroma and surface $\mathrm{pH}$ as well as ammonia aroma and salty taste (Figure 3 ). These metrics were major contributors to PC 1 and PC 2. We found a strong, highly significant positive correlation between ammonia aroma and surface $\mathrm{pH}(\mathrm{r}=0.79 ; P<$ $0.0001)$. This is an expected result, as surface ammonia production is considered a main cause of rind alkalinization (McSweeney, 2004). The relationship between ammonia aroma and salty taste showed a moderate, significant positive correlation $(\mathrm{r}=0.60 ; P=0.002)$. This indicates that ammonia aroma might have altered the perception of saltiness as perceived by the panelists. Aroma is known to influence taste perception and can increase saltiness intensity depending on the context (Lawrence et al., 2009, 2011). This could be the case for ammonia aroma and perceived saltiness of washed-rind cheeses, and this potential relationship warrants further study. 


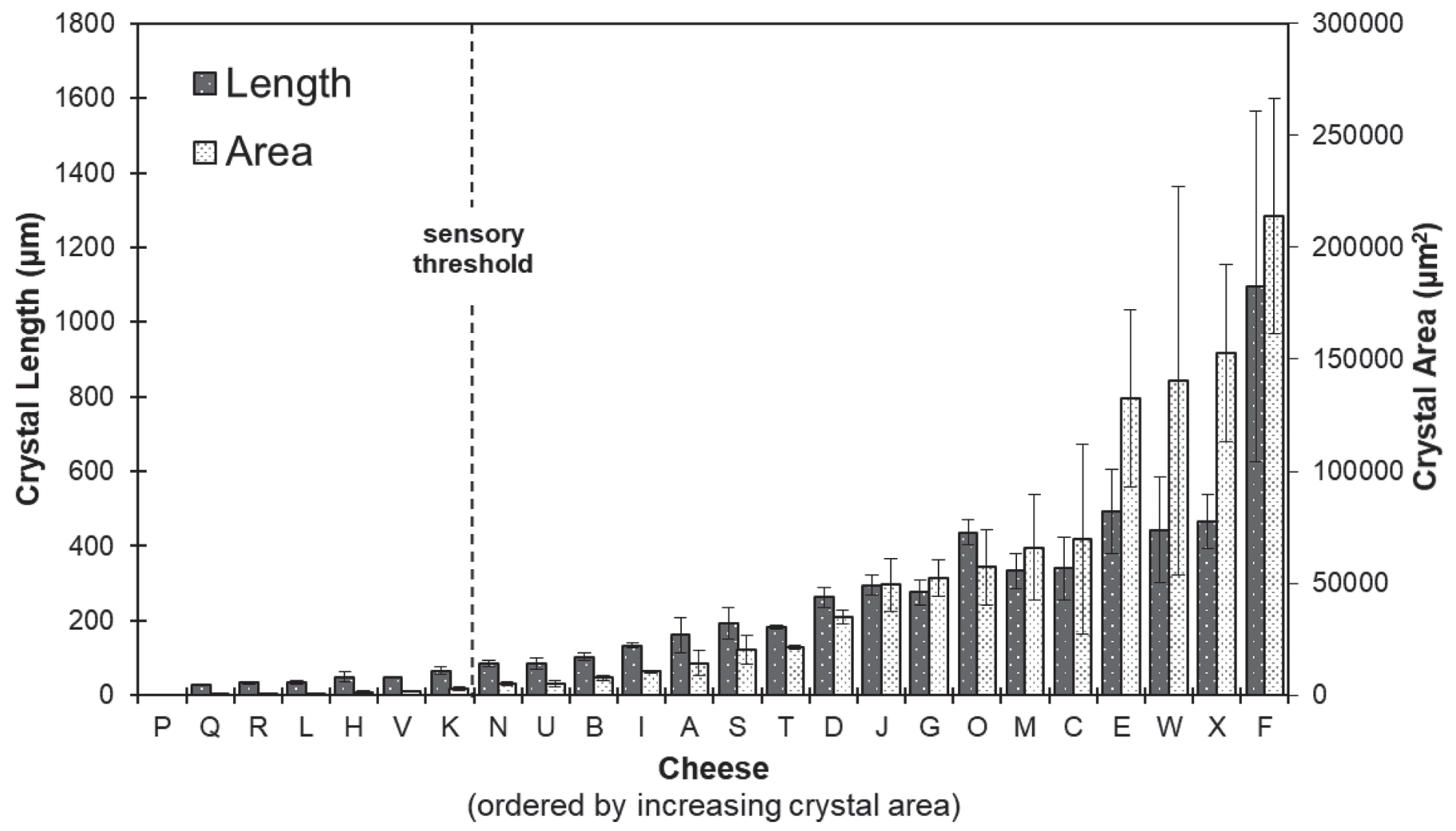

Figure 5. Mean crystal area and mean crystal length measurements for cheeses used in this study. Error bars show 1 SD from mean.

\section{Occurrence of Grittiness}

Grittiness occurring at a crystal size threshold of approximately $66 \mu \mathrm{m}$ is inline with thresholds established in other products. Grittiness in ice cream is caused by ice crystals that exceed $45 \mu \mathrm{m}$ (Goff and Hartel, 2013). Hough et al. (1990) found sandiness occurred in Dulce de Leche when lactose crystals were in the size range of $45 \mu \mathrm{m}$ to $105 \mu \mathrm{m}$, depending on the number of crystals. The sensory perception of grittiness is a complex physiological phenomenon. Particle size, shape, hardness, and viscosity of dispersion medium can influence grittiness perception (Engelen et al., 2005).

For cheeses above the grittiness threshold, grittiness was highly correlated with crystal length and crystal area (Table 5.). Pearson correlation coefficients for grittiness and crystal length and crystal area were 0.927 and 0.957 , respectively; both of these correlations were highly significant $(P<0.001)$. Of note is a higher correlation of crystal area to grittiness compared with crystal length and grittiness. This might indicate that capturing crystal size data in 2 dimensions better represents how it is perceived in a 3-dimensional context (i.e., sensory evaluation). A downside of this approach is the necessity to employ image analysis, which re- quires advanced training of personnel. One-dimensional measurements (e.g., length, diameter) can be gathered directly by the researchers using a microscope and calibrated ocular. Looking at PCA and correlation coefficients, crystal circularity was poorly associated with grittiness $(\mathrm{r}=-0.341 ; P=0.181)$. This could indicate crystal shape was not a causative factor for grittiness occurrence and crystal size played a much larger role.

A chi-squared test was employed to gauge if crystal type had a large influence on grittiness perception. The presence of each crystal type, along with grittiness occurrence, was coded in a binary format (i.e., yes or no) for each of the 23 cheeses that contained crystals. Phi-coefficients and likelihood ratio probabilities were determined for the influence of ikaite, calcite, brushite, and struvite on grittiness. Tyrosine was not used due to it occurring in only 1 sample. Each crystal type had relatively low phi-coefficients $(<0.3)$ indicating small effect sizes across the various crystal types. This could indicate that grittiness will occur regardless of crystal type and that crystal size is a larger determining factor. Understanding the dynamic relationship between crystal type and crystal growth rate would aid in determining how to control potential grittiness and warrants further study. 
Table 5. Pearson correlation coefficients and correlation probabilities (in parentheses below) of crystal size/ shape data and grittiness sensory intensity for cheeses above grittiness threshold

\begin{tabular}{lcccc}
\hline Measurement & Grittiness & Crystal length & Crystal area & Crystal circularity \\
\hline Grittiness & 1.000 & 0.927 & 0.957 & -0.341 \\
Crystal length & $(<0.001)$ & $(<0.001)$ & $(<0.001)$ & $(0.181)$ \\
& 0.927 & 1.000 & 0.922 & -0.487 \\
Crystal area & $(<0.001)$ & $(<0.001)$ & $(<0.001)$ & $(0.048)$ \\
Crystal circularity & 0.957 & 0.922 & $(<0.001)$ & $(0.264)$ \\
& $(<0.001)$ & $(<0.001)$ & -0.287 & 1.000 \\
& -0.341 & $(0.0487$ & $(0.264)$ & $(<0.001)$ \\
\hline
\end{tabular}

\section{CONCLUSIONS}

The present work indicates that surface-situated crystals are common to the soft washed-rind cheese category. These crystals induce a grittiness sensory sensation, which is largely influenced by crystal size. Cheeses that formed surface crystals and displayed perceived grittiness showed wide variation in moisture content, but we made no attempt to evaluate the effect of cheese composition on crystal size and grittiness intensity. Future research to understand the role that cheese composition and proteolysis play in the formation and growth rates of surface crystals is warranted. Future efforts to determine how crystal growth can be controlled in a deliberate fashion, and tailored to prevent grittiness, such as controlling carbon dioxide and ammonia concentrations in the headspace above the surface smear, also appear to hold promise. Furthermore, because crystals are composed of major structural minerals, manipulating their growth dynamics to modulate curd demineralization through modified atmosphere strategies could also potentially aid in controlling texture development, such as softening and liquefaction.

\section{ACKNOWLEDGMENTS}

This study was funded by USDA Hatch Project 033286. The National Science Foundation (Alexandria, VA) is acknowledged for support of grant EAR-0922961 for the purchase of the powder X-ray diffractometer. Adèle Berrier and the rest of the staff at The Cellars at Jasper Hill (Greensboro, VT) are gratefully acknowledged for their support with sensory evaluation.

\section{REFERENCES}

Agarwal, S., J. R. Powers, B. G. Swanson, S. Chen, and S. Clark. 2006. Cheese $\mathrm{pH}$, protein concentration, and formation of calcium lactate crystals. J. Dairy Sci. 89:4144-4155. https://doi.org/10.3168/ jds.S0022-0302(06)72459-6.

Altunkaynak, B. Z., M. E. Onger, M. E. Altunkaynak, E. Ayrancı, and S. Canan. 2011. A brief introduction to stereology and sampling strategies: Basic concepts of stereology. Neuroquantology 10. https://doi.org/10.14704/nq.2012.10.1.427.
Amano, K. 1950. A note on sodium phosphate crystals found in mildcured salmon. Nippon Suisan Gakkaishi 15:638-641. https://doi .org/10.2331/suisan.15.638.

Arifuzzaman, S., and S. Rohani. 2004. Experimental study of brushite precipitation. J. Cryst. Growth 267:624-634. https://doi.org/10 1016/J.JCRYSGRO.2004.04.024.

Bockelmann, W. 2011. Smear-ripened cheeses. Pages 391-401 in Encyclopedia of Dairy Sciences, 2nd ed. J. W. Fuquay, P. F. Fox, and P. L. H. McSweeney, ed. Elsevier, Academic Press, London, UK.

Brennan, N. M., T. M. Cogan, M. Loessner, and S. Scherer. 2004 Bacterial surface-ripened cheeses. Pages 199-225 in Cheese: Chemistry, Physics, and Microbiology. Vol. 2. Academic Press, Cambridge, MA.

Brennan, N. M., A. C. Ward, T. P. Beresford, P. F. Fox, M. Goodfellow, and T. M. Cogan. 2002. Biodiversity of the bacterial flora on the surface of a smear cheese. Appl. Environ. Microbiol. 68:820 830. https://doi.org/10.1128/AEM.68.2.820-830.2002.

D'Incecco, P., S. Limbo, F. Faoro, J. Hogenboom, V. Rosi, S. Morandi, and L. Pellegrino. 2016. New insight of crystal and spot development in hard and extra-hard cheeses: Association of spots with incomplete aggregation of curd granules. J. Dairy Sci. 99:6144-6156.

de Mendiburu, F. 2017. agricolae: Statistical Procedures for Agricultural Research. R package version 1.2-8. Accessed Dec. 23, 2017. https://cran.r-project.org/web/packages/agricolae/index.html.

Dugat-Bony, E., L. Garnier, J. Denonfoux, S. Ferreira, A.-S. Sarthou P. Bonnarme, and F. Irlinger. 2016. Highlighting the microbial diversity of 12 French cheese varieties. Int. J. Food Microbiol. 238:265-273. https://doi.org/10.1016/j.ijfoodmicro.2016.09.026.

Engelen, L., R. A. de Wijk, A. van der Bilt, J. F. Prinz, A. M. Janssen, and F. Bosman. 2005. Relating particles and texture perception. Physiol. Behav. 86:111-117. https://doi.org/10.1016/j.physbeh .2005.06.022.

Goff, H. D., and R. W. Hartel. 2013. Ice Cream. 7th ed. Springer, Berlin, Germany.

Gori, K., H. D. Mortensen, N. Arneborg, and L. Jespersen. 2007. Ammonia production and its possible role as a mediator of communication for Debaryomyces hansenii and other cheese-relevant yeast species. J. Dairy Sci. 90:5032-5041. https://doi.org/10.3168/jds 2006-750.

Hélias, A., P. Mirade, and G. Corrieu. 2007. Modeling of Camemberttype cheese mass loss in a ripening chamber: Main biological and physical phenomena. J. Dairy Sci. 90:5324-5333. https://doi.org/ 10.3168/jds.2007-0272.

Hitchcock, D. I. 1924. The solubility of tyrosine in acid and in alkali. J. Gen. Physiol. 6:747-757.

Hough, G., E. Martinez, and A. Contarini. 1990. Sensory and objective measurement of sandiness in Dulce de Leche, a typical argentine dairy product. J. Dairy Sci. 73:604-611. https://doi.org/10.3168/ jds.S0022-0302(90)78709-7.

Hu, Y.-B., D. A. Wolf-Gladrow, G. S. Dieckmann, C. Volker, and G. Dehrke. 2014. A laboratory study of ikaite $\left(\mathrm{CaCO}_{3} \cdot 6 \mathrm{H}_{2} \mathrm{O}\right)$ precipitation as a function of $\mathrm{pH}$, salinity, temperature and phosphate concentration. Mar. Chem. 162:10-18. https://doi.org/10.1016/J .MARCHEM.2014.02.003.

Imai, E., K. Hatae, and A. Shimada. 1995. Oral perception of grittiness: Effect of particle size and concentration of the dispersed 
particles and the dispersion medium. J. Texture Stud. 26:561-576. https://doi.org/10.1111/j.1745-4603.1995.tb00804.x.

Johnson, M. E., B. A. Riesterer, C. Chen, B. Tricomi, and N. F. Olson. 1990. Effect of packaging and storage conditions on calcium lactate crystallization on the surface of cheddar cheese. J. Dairy Sci. 73:3033-3041. https://doi.org/10.3168/jds.S0022-0302(90)78990 -4 .

Karahadian, C., and R. C. Lindsay. 1987. Integrated roles of lactate, ammonia, and calcium in texture development of mold surfaceripened cheese. J. Dairy Sci. 70:909-918. https://doi.org/10.3168/ jds.S0022-0302(87)80094-2.

Kim, D., K. J. Min, K. Lee, M. S. Yu, and K. Y. Park. 2016. Effects of $\mathrm{pH}$, molar ratios and pre-treatment on phosphorus recovery through struvite crystallization from effluent of anaerobically digested swine wastewater. Environ. Eng. Res. 22:12-18. https://doi .org/10.4491/eer.2016.037.

Lawrence, G., C. Salles, O. Palicki, C. Septier, J. Busch, and T. Thomas-Danguin. 2011. Using cross-modal interactions to counterbalance salt reduction in solid foods. Int. Dairy J. 21:103-110. https: //doi.org/10.1016/J.IDAIRYJ.2010.09.005.

Lawrence, G., C. Salles, C. Septier, J. Busch, and T. Thomas-Danguin. 2009. Odour-taste interactions: A way to enhance saltiness in low-salt content solutions. Food Qual. Prefer. 20:241-248. https: //doi.org/10.1016/J.FOODQUAL.2008.10.004.

Lê, S., J. Josse, and F. Husson. 2008. FactoMineR: An R package for multivariate analysis. J. Stat. Softw. 25:1-18. https://doi.org/10 $.18637 /$ jss.v025.i01.

Le Graet, Y., and G. Brule. 1988. Migration des macro et oligo-éléments dans un fromage à pâte molle de type Camembert. Lait 68:219-234. https://doi.org/10.1051/lait:1988215.

Le Graet, Y., A. Lepienne, G. Brule, and P. Ducruet. 1983. Migration du calcium et des phosphates inorganiques dans les fromages à pâte molle de type Camembert au cours de l'affinage. Lait 63:317332. https://doi.org/10.1051/lait:1983629-63019.

Le Mens, P., P. Parguel, H. Tormo, Y. Lefrileux, G. Bourge, and C. Guillard. 2000. Description sensorielle de trois fromages fermiers: Le Banon, la Brique du Forez et le Rogeret. Renc. Rech. Ruminants 7:300-303.

Li, C. H., and C. K. Lee. 1993. Minimum cross entropy thresholding. Pattern Recognit. 26:617-625. https://doi.org/10.1016/0031 -3203(93)90115-D.

Lucey, J. A., and P. F. Fox. 1993. Importance of calcium and phosphate in cheese manufacture: A review. J. Dairy Sci. 76:1714-1724. https://doi.org/10.3168/jds.S0022-0302(93)77504-9.

McSweeney, P. L. H. 2004. Biochemistry of cheese ripening. Int. J. Dairy Technol. 57:127-144. https://doi.org/10.1111/j.1471-0307 .2004.00147.x.

Meilgaard, M., G. V. Civille, and B. T. Carr. 2007. Sensory Evaluation Techniques. Taylor \& Francis, Abingdon, UK.

Meyer, D., A. Zeileis, and K. Hornik. 2017. vcd: Visualizing Categorical Data. R package version 1.4-4. Accessed Dec. 23, 2017. https:/ /cran.r-project.org/web/packages/vcd/index.html.

Picque, D., M.-N. Leclercq-Perlat, and G. Corrieu. 2006. Effects of atmospheric composition on respiratory behavior, weight loss, and appearance of camembert-type cheeses during chamber ripening. J. Dairy Sci. 89:3250-3259. https://doi.org/10.3168/jds.S0022 $-0302(06) 72600-5$.
Polowsky, P. J., G. F. Tansman, P. S. Kindstedt, and J. M. Hughes, 2018. Characterization and presumptive identification of surface crystals on smear ripened cheese by polarized light microscopy. J. Dairy Sci. 101:7714-7723. https://doi.org/10.3168/jds.2018-14712.

R Core Team. 2016. R: A language and environment for statistical computing. R Foundation for Statistical Computing, Vienna, Austria.

Riahi, M. H., I. C. Trelea, M.-N. Leclercq-Perlat, D. Picque, and G. Corrieu. 2007. Model for changes in weight and dry matter during the ripening of a smear soft cheese under controlled temperature and relative humidity. Int. Dairy J. 17:946-953. https://doi.org/10 .1016/J.IDAIRYJ.2006.11.002.

Rysgaard, S., R. N. Glud, K. Lennert, M. Cooper, N. Halden, R. J. G. Leakey, F. C. Hawthorne, and D. Barber. 2012. Ikaite crystals in melting sea ice - I mplications for pCO 2 and pH levels in Arctic surface waters. The Cryosphere 6:901-908. https://doi.org/10 $.5194 /$ tc-6-901-2012.

Schindelin, J., I. Arganda-Carreras, E. Frise, V. Kaynig, M. Longair T. Pietzsch, S. Preibisch, C. Rueden, S. Saalfeld, B. Schmid, J.-Y Tinevez, D. J. White, V. Hartenstein, K. Eliceiri, P. Tomancak, and A. Cardona. 2012. Fiji: An open-source platform for biological-image analysis. Nat. Methods 9:676-682. https://doi.org/10 $.1038 /$ nmeth.2019.

Tansman, G. F., P. S. Kindstedt, and J. M. Hughes. 2015. Crystal fingerprinting: elucidating the crystals of Cheddar, Parmigiano-Reggiano, Gouda, and soft washed-rind cheeses using powder X-ray diffractometry. Dairy Sci. Technol. 95:651-664. https://doi.org/10 .1007/s13594-015-0225-6.

Tansman, G. F., P. S. Kindstedt, and J. M. Hughes. 2017a. Crystallization and demineralization phenomena in washed-rind cheese. J. Dairy Sci. 100:8964-8704. https://doi.org/10.3168/jds.2017-13067.

Tansman, G. F., P. S. Kindstedt, and J. M. Hughes. 2017b. Crystallization and demineralization phenomena in stabilized white mold cheese. J. Dairy Sci. 100:6074-6083. https://doi.org/10.3168/jds .2016-12259.

Tansman, G. F., P. S. Kindstedt, and J. M. Hughes. 2017c. Minerals in food: Crystal structures of ikaite and struvite from bacterial smears on washed-rind cheese. Can. Mineral. 55:89-100. https:// doi.org/10.3749/canmin.1600038.

Vaisey-Genser, M., B. K. Vane, and S. Johnson. 1989. Graininess, crystal size, and firmness of stored canola oil margarines. J. Texture Stud. 20:347-361. https://doi.org/10.1111/j.1745-4603.1989 .tb00445.x.

Wilsenach, J. A., C. A. H. Schuurbiers, and M. C. M. van Loosdrecht. 2007. Phosphate and potassium recovery from source separated urine through struvite precipitation. Water Res. 41:458-466. https: //doi.org/10.1016/j.watres.2006.10.014.

Wolfe, B. E., J. E. Button, M. Santarelli, and R. J. Dutton. 2014. Cheese rind communities provide tractable systems for in situ and in vitro studies of microbial diversity. Cell 158:422-433. https:// doi.org/10.1016/j.cell.2014.05.041.

Wu, Q., P. L. Bishop, and T. C. Keener. 2005. A strategy for controlling deposition of struvite in municipal wastewater treatment plants. Water Environ. Res. 77:199-207. https://doi.org/10.2175/ $106143005 X 41771$. 